\title{
NONLINEAR VIBRATION OF A PENDULUM WITH A SUPPORT IN HARMONIC MOTION
}

\author{
NGUYen VAN DAO \\ Institute of Mechanics
}

(This paper as been published in:

Proceedings of the Fifth National Conference on Mechanics, Vol. 1 (38-45), 1993)

\begin{abstract}
The nonlinear vibration of a pendulum whose support undergoes arbitrary rectilinear harmonic motion is studied. The main attention is paid to the resonant cases and the stationary vibrations. The resonant conditions are explained. The amplitude frequency curves are plotted for various values of parameters and the stability of vibration is investigated. The rotating motion of the pendulum and its stability are also considered.
\end{abstract}

\section{EQUATION OF MOTION}

Let us consider the vibration of a pendulum consisting of a negligible weight rod AM of length and a load $M$ of mass $m$. The pendulum support undergoes rectilinear harmonic motion by means of a mechanism shown in Fig. 1 when the crank ON of length $R$ rotates around $\mathrm{O}$ with a constant angular velocity $\Omega$ and translates slotted bar BA of length $L$ along slides 1.1. We shall take the origin of the $y$ axis vertically up. The position of the pendulum will be specified by angle so that AM makes with vertical axis (Fig. 1). The kinetic and potential energies of the pendulum, $T$ and $V$ respectively are:

$$
\begin{aligned}
T & =\frac{m}{2}\left[\ell^{2} \dot{\varphi}^{2}+R^{2} \Omega^{2} \sin ^{2} \Omega t-2 R \Omega \ell \dot{\varphi} \sin \Omega t \sin (\delta+\varphi)\right], \\
V & =m g[(L+R \cos \Omega t) \cos \delta-\ell \cos \varphi] .
\end{aligned}
$$

Using Lagrange's equation, taking into account the damping force, the equation of motion of the pendulum is obtained as

$$
\ddot{\varphi}+\omega^{2} \sin \varphi+h \dot{\varphi}-\frac{R}{\ell} \Omega^{2} \cos \Omega t \sin (\delta+\varphi)=0,
$$

where $\omega^{2}=g / \ell, h$ is the damping coefficient.

We assume that $R / \ell$ and $h$ are small and we shall consider small vibrations of the pendulum about the vertical axis, so that $\sin \varphi \approx \varphi-\left(\varphi^{3} / 6\right), \cos \varphi \approx 1-\left(\varphi^{2} / 2\right)$. The smallness of the mentioned quantities can be taken into consideration by introducing a small dimensionless parameter $\ell$ which will be set equal to unity in the final results. Thus, we are led to consider the following equation of motion:

$$
\varphi^{\prime \prime}+\varphi=\varepsilon\left[-h_{1} \varphi^{\prime}+\frac{1}{6} \varphi^{3}+C \gamma^{2} \cos \gamma \tau+D \gamma^{2} \cdot \varphi \cos \gamma \tau\right],
$$




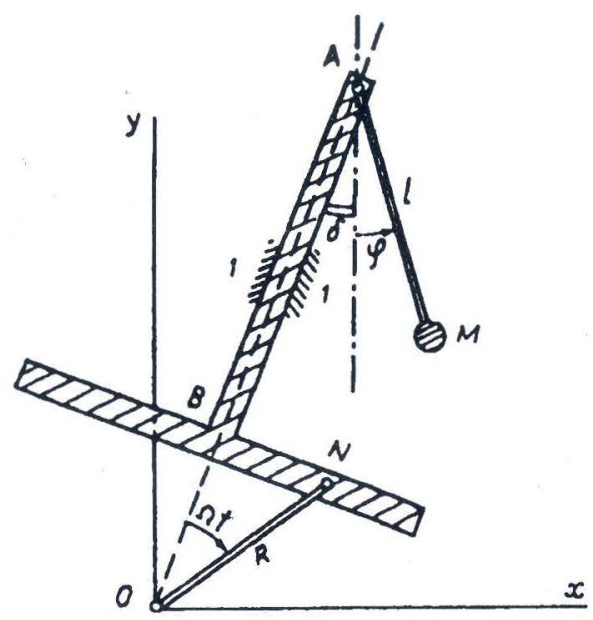

Fig. 1

where

$$
\tau=\omega t, \quad C=\frac{R}{\ell} \sin \delta, \quad D=\frac{R}{\ell} \cos \delta, \quad h_{1}=\frac{h}{\omega}, \quad \gamma=\frac{\Omega}{\omega}
$$

and a prime denotes the derivative with respect to the dimensionless time $\tau$.

In the following sections two resonant cases which cause intensive growth of the amplitude of vibration of the pendulum will be studied.

\section{PRINCIPAL RESONANCE}

We consider the cases when $\gamma$ differs a little from unity. We are interested in finding out what happens close to resonance, that is to say when $\gamma^{2}-1$ is small, namely:

$$
\gamma^{2}=1+\frac{\varepsilon \Delta}{\omega^{2}}
$$

where $\Delta$ is a detuning parameter.

Let us introduce in equation (1.3) the variables $a$ and $\eta$ as follows

$$
\varphi=a \cos \theta, \quad \varphi^{\prime}=-a \gamma \sin \theta, \quad \theta=\gamma \tau+\eta
$$

here the condition

$$
a^{\prime} \cos \theta-a \eta^{\prime} \sin \theta=0
$$

is imposed. The equations for new variables will be

$$
\begin{aligned}
\gamma a^{\prime} & =-\varepsilon\left(\frac{\Delta}{\omega^{2}} \cdot \varphi+f\right) \sin \theta \\
\gamma a \eta^{\prime} & =-\varepsilon\left(\frac{\Delta}{\omega^{2}} \cdot \varphi+f\right) \cos \theta
\end{aligned}
$$

whicvh is a set of equations in standard form with

$$
f=h_{1} a \gamma \sin \theta+\frac{1}{6} a^{3} \cos ^{3} \theta+C \gamma^{2} \cos \gamma \tau+D \gamma^{2} a \cos \theta \cdot \cos \gamma \tau
$$


In the first approximation the right-hand sides of (2.4) may be replaced by their mean values, regarding $a$ and $\eta$ as constants [1]:

$$
\begin{aligned}
\gamma a^{\prime} & =-\frac{\varepsilon}{2}\left(h_{1} \gamma a+C \gamma^{2} \sin \eta\right), \\
\gamma a \eta^{\prime} & =-\frac{\varepsilon}{2}\left(\frac{\Delta}{\omega^{2}} a+\frac{1}{8} a^{3}+C \gamma^{2} \cos \eta\right) .
\end{aligned}
$$

The stationary amplitude $a_{0}$ and phase $\eta_{0}$ are determined by

$$
\begin{aligned}
h_{1} \gamma a_{0}+C \gamma^{2} \sin \eta_{0} & =0, \\
\frac{\Delta}{\omega^{2}} a_{0}+\frac{1}{8} a_{0}^{3}+C \gamma^{2} \cos \eta_{0} & =0 .
\end{aligned}
$$

A simple calculation eliminating $\eta$ leads to the response curve equation:

$$
\begin{aligned}
& W\left(a_{0}, \gamma^{2}\right)=0, \\
& W=a_{0}^{2}\left[h_{1}^{2} \gamma^{2}+\left(\gamma^{2}-1+\frac{1}{8} a_{0}^{2}\right)^{2}\right]-C^{2} \gamma^{4} .
\end{aligned}
$$

This relation is ploted in Fig. 2 for the parameters:

$R=2 \mathrm{~cm}, \ell=100 \mathrm{~cm}, \delta=0.78 \mathrm{rad}, g=980 \mathrm{~cm} / \mathrm{sec}^{2}, h=2.47 \cdot h_{1}, \omega=\sqrt{g / \ell}=2.47$, $\omega^{2}=6.125, C=8.8 \cdot 10^{-3} \cdot 1 . h_{1}=10^{-2}$ and $2 . h_{1}=4 \cdot 10^{-2}$.

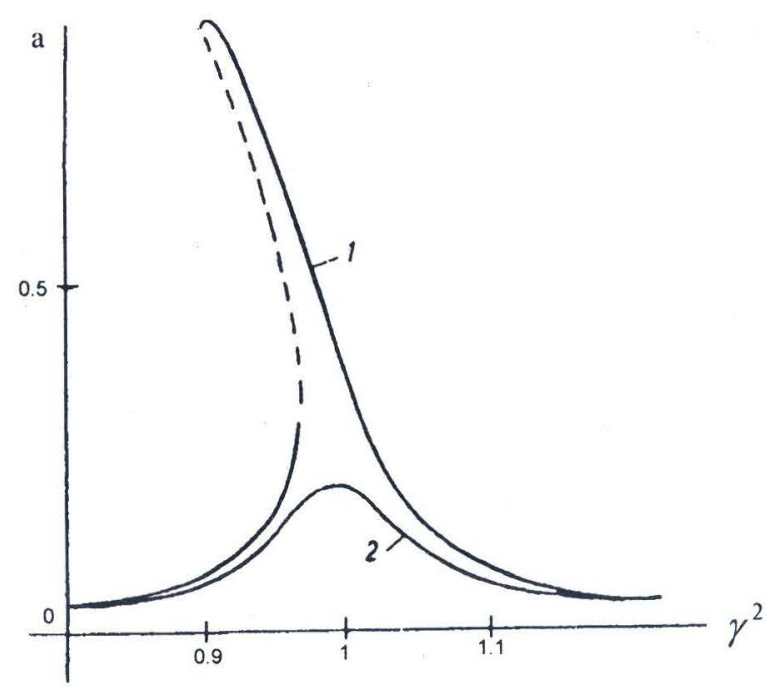

Fig. 2

Let us now discuss the stability of the possible stationary regimes. To do this we study the eigenvalues of the matrix of coefficients of variational equations of (2.6):

$$
\left(\begin{array}{cc}
-\frac{\varepsilon}{2} h_{1} \gamma & -\frac{\varepsilon C}{2} \gamma^{2} \cos \eta_{0} \\
-\frac{\varepsilon}{2}\left(\frac{\Delta}{\omega^{2}}+\frac{3}{8} a_{0}^{2}\right) & \frac{\varepsilon C}{2} \gamma^{2} \sin \eta_{0}
\end{array}\right)
$$


The equation defining these eigenvalue is

$$
a_{0} \gamma^{2} \lambda^{2}+\varepsilon \gamma^{2} a_{0} h_{1} \lambda+\frac{\varepsilon^{2}}{8} \frac{\partial W}{\partial a_{0}}=0
$$

from which we obtain the condition for asymptotic stability

$$
\frac{\partial W}{\partial a_{0}}>0
$$

It is noted that function $W\left(a_{0}, \gamma^{2}\right)$ is positive (negative) outside (inside) of resonant curve and equal to zero on it. So, condition (2.11) shows that the upper branches of resonant curves (heavy lines) in Fig. 2 correspond to stable stationary regimes and the broken lines to unstable ones.

\section{PARAMETRIC RESONANCE}

It is supported that $\gamma$ is approximately equal to 2 , namely

$$
\gamma^{2}=4\left(1+\frac{\varepsilon}{\omega^{2}} \Delta_{1}\right)
$$

The solution of equation (1.3) in this cases is found to be

$$
\varphi=b \cos \left(\frac{\gamma}{2} r+\alpha\right), \quad \varphi^{\prime}=-\frac{b}{2} \gamma \sin \left(\frac{\gamma}{2} r+\alpha\right) .
$$

In the first approximation (1.3) can be replaced by the averaged ones:

$$
\begin{aligned}
\gamma b^{\prime} & =-\frac{\varepsilon}{2} b \gamma\left(h_{1}+D \gamma \sin 2 \alpha\right), \\
\gamma b \alpha^{\prime} & =-\frac{\varepsilon}{2} b\left(\frac{2 \Delta_{1}}{\omega^{2}}+\frac{1}{4} b^{2}+D \gamma^{2} \cos 2 \alpha\right) .
\end{aligned}
$$

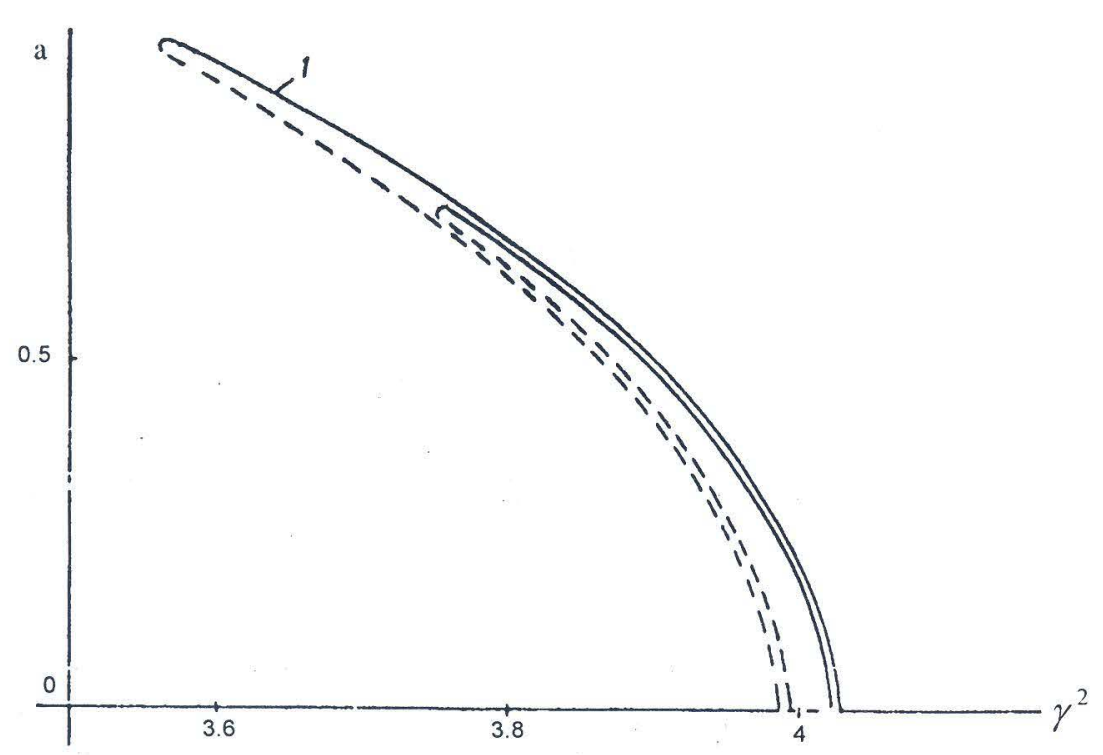

Fig. 3 
It is clear that $b=0$ is a solution of equations (3.3), but as we shall show later, it may happen that this solution is unstable and that the system begins to vibrate spontaneously. The stationary amplitude $b_{0}$ is determined from equation $b^{\prime}=\alpha^{\prime}=0$ by elimination $\alpha$ :

$$
b^{2}=8\left(1-\frac{\gamma^{2}}{4}\right) \pm 4 \gamma \sqrt{D^{2} \gamma^{2}-h_{1}^{2}} .
$$

The response curves are ploted in Fig. 3 for $D=8.8 \cdot 10^{-2}$ and 1) $h_{1}=1.65 \cdot 10^{-2}, 2$ ) $h_{1}=1.7 \cdot 10^{-2}$. The study shows that only the positive sign before the radical (3.4) corresponds to the asymptotical stability of nontrivial stationary vibration; and that the solution $b=0$ is stable outside the resonant curve and unstable inside it. Thus, only the heavy line (Fig. 3) of the resonant curve correspond to stability of vibration.

\section{ROTATING MOTION OF THE PENDULUM}

Assuming that $h$ and $R / \ell$ are small we consider the rotating motion of the pendulum governed by equation:

$$
\ddot{\varphi}+\omega^{2} \sin \varphi=\varepsilon F(\varphi, \dot{\varphi}, t)
$$

where

$$
F(\varphi, \dot{\varphi}, t)=-h \dot{\varphi}-\frac{R}{\ell} \Omega^{2} \cos \Omega t \sin (\delta+\varphi) .
$$

It is supposed that the energy of the system considered is high so that when $\varepsilon=0$ the pendulum will be rotating (equation (4.1)).

We introduce the variable $a$ and $\psi[2]$ :

$$
\begin{aligned}
& \varphi=\xi+a \sin \xi, \\
& \dot{\varphi}=\nu(a)+a \nu(a) \cos \xi,
\end{aligned}
$$

where

$$
\xi=\Omega t+\psi, \quad \nu(a)=1 / \sqrt{a}, \quad \dot{\xi}=\nu(a)
$$

and $\varphi$ is the solution of the degenerate equation $\varepsilon=0$ if $a$ and $\psi$ are constants; so that

$$
-a \nu^{2}(a) \sin \xi+\omega^{2} \sin \varphi \equiv 0 .
$$

Equation (4.3) imply that

$$
\dot{\xi}(1+a \cos \xi)+\dot{a} \sin \xi-\nu(a)(1+a \cos \xi)=0 .
$$

The second equation for $\dot{\xi}$ and $\dot{a}$ is obtained by substituting equation (4.3) into equation (4.1):

$$
-a \nu \dot{\xi} \sin \xi+\left[\nu_{a}(1+a \cos \xi)+\nu \cos \xi\right] \dot{a}+\omega^{2} \sin \varphi=\varepsilon F(\varphi, \dot{\varphi}, t)
$$

here the subscript " $a$ " or ()$_{a}$ denotes the derivative with respect to the amplitude $a$. From these equations we get:

$$
\begin{aligned}
& \dot{a}=-\frac{\varepsilon}{\Delta} F(\varphi, \dot{\varphi}, t)(1+a \cos \xi), \\
& \dot{\psi}=\nu(a)-\Omega+\frac{\varepsilon}{\Delta} \cdot F(\varphi, \dot{\varphi}, t) \sin \xi,
\end{aligned}
$$


where

$$
-\Delta=a \nu \sin ^{2} \xi+(1+a \cos \xi)\left[\nu_{a}(1+a \cos \xi)+\nu \cos \xi\right] .
$$

Substituting here $\nu=1 / \sqrt{a}$ we have

$$
\frac{1}{\Delta}=2 a \sqrt{a}+0\left(\sqrt{a} \cdot a^{3}\right) .
$$

We shall consider the principal resonant case when the amplitude a takes values close to $a_{0}$ determined by

$$
\Omega \approx \nu\left(a_{0}\right)=\frac{1}{\sqrt{a_{0}}}
$$

and use the Jacobie expansions of trigonometric functions in Bessel functions [3]:

$$
\begin{aligned}
\sin (a \sin \xi) & =2 \sum_{n=1}^{\infty} J_{2 n-1}(a) \sin (2 n-1) \xi, \\
\cos (a \sin \xi) & =J_{0}(a)+2 \sum_{n=1}^{\infty} J_{2 n}(a) \cos 2 n \xi \\
J_{m}(a) & =\sum_{k=0}^{\infty} \frac{(-1)^{k}}{k !(m+k) !}\left(\frac{a}{2}\right)^{m+2 k}, \quad m=0,1,2, \ldots
\end{aligned}
$$

Limiting by considering the vibration with small amplitude $a$ we have in the first approximation averaged equations of the form:

$$
\begin{aligned}
& \dot{a}=\frac{\varepsilon}{\Delta} h \nu(a)\left(1+\frac{a^{2}}{2}\right)-\frac{\varepsilon}{\Delta} \frac{R}{\ell} \Omega^{2}\left(\alpha_{1} \cos \psi+\alpha_{2} \sin \psi\right), \\
& \dot{\psi}=\nu(a-\Omega)+\frac{\varepsilon}{\Delta} \cdot \frac{R}{\ell} \Omega^{2}\left(\alpha_{3} \cos \psi+\alpha_{4} \sin \psi\right),
\end{aligned}
$$

where

$$
\begin{aligned}
& \alpha_{1}=\left[\frac{1}{2}\left(J_{0}+J_{2}\right)-\frac{\alpha}{4}\left(J_{1}+J_{3}\right)\right] \sin \delta, \\
& \alpha_{2}=\left[\frac{1}{2}\left(J_{0}-J_{2}\right)+\frac{\alpha}{4}\left(J_{1}+J_{3}\right)\right] \cos \delta, \\
& \alpha_{3}=\frac{1}{4}\left(J_{1}+J_{3}\right) \cos \delta, \\
& \alpha_{4}=\frac{1}{4}\left(J_{3}-3 J_{1}\right) \sin \delta, \\
& J_{0}=1-\left(\frac{a}{2}\right)^{2}, \quad J_{1}=\frac{a}{2}, \quad J_{2}=\frac{1}{2}\left(\frac{a}{2}\right)^{2}, \quad J_{3}=0 .
\end{aligned}
$$

Stationary regimes of resonant vibrations $a_{*}$ and $\psi_{*}$ are determined by equations $\dot{a}=\dot{\psi}=$ 0 :

$$
\begin{aligned}
\sqrt{a_{*}} \frac{R}{\ell} \Omega^{2}\left(\alpha_{1}^{*} \cos \psi_{*}+\alpha_{2}^{*} \sin \psi_{*}\right) & =h\left(1+\frac{a_{*}^{2}}{2}\right), \\
2 \varepsilon a_{*} \sqrt{a_{*}} \frac{R}{\ell} \Omega^{2}\left(\alpha_{3}^{*} \cos \psi_{*}+\alpha_{4}^{*} \sin \psi_{*}\right) & =\Omega-\frac{1}{\sqrt{a_{*}}},
\end{aligned}
$$




$$
\alpha_{i}^{*}=\alpha_{i}\left(a=a_{*}\right), \quad a_{*} \neq 0, \quad \Omega=\frac{1}{\sqrt{a_{0}}}=\text { const. }
$$

The solution of these equation are found in the series:

$$
\begin{aligned}
& a_{*}=a_{0}+\varepsilon a_{1}+0\left(\varepsilon^{2}\right), \\
& \psi_{*}=\psi_{0}+\varepsilon \psi_{1}+0\left(\varepsilon^{2}\right),
\end{aligned}
$$

where $a_{0}$ satisfies relation (4.10). Substituting equations (4.15) into equations (4.14) we have:

$$
\begin{aligned}
& \sqrt{a_{0}} \cdot \frac{R}{\ell} \Omega^{2}\left(\alpha_{10} \cos \psi_{0}+\alpha_{20} \sin \psi_{0}\right)=h\left(1+\frac{a_{0}^{2}}{2}\right), \\
& a_{*}=a_{0}+\varepsilon a_{1}=a_{0}+4 \varepsilon a_{0}^{3} \Omega^{2} \frac{R}{\ell}\left(\alpha_{30} \cos \psi_{0}+\alpha_{40} \sin \psi_{0}\right),
\end{aligned}
$$

here $\alpha_{i 0}=\alpha_{i}\left(a=a_{0}\right)$. The first equation of (4.16) gives the phase displacement. Then, the correction $\varepsilon a_{1}$ to the stationary amplitude $a_{0}$ will be found.

We consider now the stability of stationary solution $a_{*}$ and $\psi_{*}$ determined by formulae (4.14). For this purpose we write the variational equations for system (4.12). Let

$$
a=a_{*}+\delta a, \quad \psi=\psi_{*}+\delta \psi .
$$

By putting these expressions into (4.12) and linearizing relative to $\delta a$ and $\delta \psi$ we obtain:

$$
\begin{aligned}
\frac{d}{d t} \delta a= & \varepsilon\left\{\left(\frac{H}{\Delta}\right)_{a}-\frac{R}{\ell} \Omega^{2}\left[\left(\frac{\alpha_{1}}{\Delta}\right)_{a} \cos \psi_{*}+\left(\frac{\alpha_{2}}{\Delta}\right)_{a} \sin \psi_{*}\right]\right\} \delta a \\
& -\frac{\varepsilon}{\Delta} \frac{R}{\ell} \Omega^{2}\left(-\alpha_{1} \sin \psi_{*}+\alpha_{2} \cos \psi_{*}\right) \delta \psi \\
\frac{d}{d t} \delta \psi= & \left\{L_{a}+\varepsilon \frac{R}{\ell} \Omega^{2}\left[\left(\frac{\alpha_{3}}{\Delta}\right)_{a} \cos \psi_{*}+\left(\frac{\alpha_{4}}{\Delta}\right)_{a} \sin \psi_{*}\right]\right\} \delta a \\
& +\frac{\varepsilon}{\Delta} \frac{R}{\ell} \Omega^{2}\left(-\alpha_{3} \sin \psi_{*}+\alpha_{4} \cos \psi_{*}\right) \delta \psi
\end{aligned}
$$

where

$$
H=h \nu(a)\left(1+\frac{a^{2}}{2}\right), \quad L(a)=\nu(a)-\Omega .
$$

The characteristic equation of this system is of the form

$$
\lambda^{2}+K \lambda+G=0
$$

where

$$
\begin{aligned}
& K=\varepsilon \frac{R}{\ell} \Omega^{2}\left\{\left[\left(\frac{\alpha_{1}}{\Delta}\right)_{a}-\left(\frac{\alpha_{4}}{\Delta}\right)\right] \cos \psi_{*}+\left[\left(\frac{\alpha_{2}}{\Delta}\right)_{a}+\left(\frac{\alpha_{3}}{\Delta}\right)\right] \sin \psi\right\}-\varepsilon\left(\frac{H}{\Delta}\right)_{a}, \\
& G=\frac{\varepsilon}{\Delta} \frac{R}{\ell} \Omega^{2} L_{a}\left(a_{*}\right)\left(-\alpha_{1} \sin \psi_{*}+\alpha_{2} \cos \psi_{*}\right)+0\left(\varepsilon^{2}\right),
\end{aligned}
$$

and a prime denotes a derivative to $a_{*}$. The stability conditions will be

$$
K>0, \quad G>0 .
$$


As an example let us consider the case $\delta=0$. Then

$$
\alpha_{1}=\alpha_{4}=0, \quad \alpha_{2}=\frac{1}{2}\left(1-\frac{a^{2}}{8}\right), \quad a_{3}=\frac{a}{8},
$$

and equation (4.16) are of the form:

$$
\begin{aligned}
& \frac{R}{\ell} \Omega^{2} \sqrt{a_{0}}\left(1-\frac{a_{0}^{2}}{8}\right) \sin \omega_{0}=2 h\left(1+\frac{a_{0}^{2}}{8}\right), \\
& a_{*}=a_{0}+\frac{1}{2} \varepsilon \frac{R}{\ell} \Omega^{2} \cdot a_{0}^{1} \cos \omega_{0}, \quad a_{0}=\frac{1}{\Omega^{2}} .
\end{aligned}
$$

Because $a$ is small, the first equation of (4.22) shows that there are two values of $\psi_{0}$ lying on the first and sccond quadrants corresponding to two values of cos $\psi^{\prime} 0$ with opposite signs. Therefore the one of stationary amplitude corresponding to oos $\langle 0>0$ is large than $a_{0}$ and the other corresponding to $\cos \psi_{0}<0$ is smaller than $a_{0}$. The expressions (4.2(0) now are:

$$
\begin{aligned}
& K=h+O\left(a^{2}\right), \\
& G=-\varepsilon \frac{R}{2 \ell} \Omega^{2} \cos \psi_{0}+O\left(\varepsilon^{2}\right),
\end{aligned}
$$

and the stability condition gives:

$$
\cos \psi_{0}<0
$$

For the cases $\delta=\pi / 2$ we have

$$
\begin{aligned}
& \alpha_{2}=\alpha_{3}=0, \quad \alpha_{1}=\frac{1}{2}+0(a), \quad \alpha_{4}=-\frac{3 a}{8}+0(a), \\
& a_{*}=a_{0}+\varepsilon a_{1}=a_{0}-\frac{3}{2} \varepsilon \frac{R}{\ell} \Omega^{2} a_{0}^{4} \sin \psi_{0}, \\
& K=\varepsilon h+0\left(a^{2}\right), \\
& G=\frac{\varepsilon}{2 \ell} R \Omega^{2} \sin \psi_{0}+0\left(\varepsilon^{2}\right),
\end{aligned}
$$

and the stability condition is

$$
\sin \psi_{0}>0 \text {. }
$$

So, in both cases $(\delta=0$ and $\delta=\pi / 2)$ the stationary vibration with small amplitude is stable and that with large amplitude is unstable (see equations (4.22) and equations (4.24). (4.25) and (4.26)).

\section{CONCLUSION}

1. The stationary nonlinear vibrations of the pendulum and its stability have been considered.

2. To avoid resonance of the pendulum, the parameters of the system considered should be chosen so that $\omega^{2}$ difers from $\Omega^{2}$ and $\frac{1}{4} \Omega^{2}$ or

$$
\frac{g}{t}+\Omega^{2}, \frac{1}{4} \Omega^{2}
$$


3. The rotating motion of the pendulum may occur. Using the averaging method of nonlinear mechanics and the Jacobie expansions, the small "vibration" of the pendulum around the stationary rotation and its stability have been studied.

\section{REFERENCES}

1. N. N. Bogoliubov, Yu. A. Mitropolskii, Asymptotic Methods in Nonlinear Vibrations, Moscow 1974 (in Russian).

2. N. N. Moiseev, Asymptotic Methods of Nonlinear Mechanics, Moscow 1981 (in Russian).

3. V. I. Smirnov, Cours of High Mathematics, tom 3, part 2, Moscow 1956 (in Russian).

\section{DAO ĐộNG PHI TUYẾN CƯA CON LẮC CÓ ĐIỂM TREO CHUYỄN ĐộNG DIỀU HOÀ}

Dao động phi tuyến của con lắc có điềm treo thực hiện chuyển động điều hoà được nghiên cứu. Điều quan tâm chính là các trường hợp cộng hường và dao động bình ồn. Các điều kiẹn ờn định sẽ được trình bầy chi tiết. Đường cong biên độ - tần số được vẽ với các giá trị khác nhau cua tham số. Sự ơn định của dao động được khảo sát. Chuyển động quay tròn của con lắc và sự ôn định tương ứng cũng được xem xét. 